\title{
Sarcome myofibroblastique de bas grade : encore un cas sur la langue
}

\section{Low grade myofibroblastic sarcoma: a new case in the tongue}

\author{
NORA ALNO', GILBERT DE MELLO ${ }^{1}$, FABRICE CLIPET ${ }^{1}$, PLINIO RONDI², TOMMASO LOMBARDI ${ }^{3}$, JACKY SAMSON²
}

\section{RÉSUMÉ}

Le sarcome myofibroblastique est une tumeur maligne mésenchymateuse extrêmement rare, de description récente, qui peut se développer dans tous les tissus, mais le plus souvent dans les tissus mous de la tête et du cou. Cette tumeur, qui a une évolution relativement asymptomatique, récidive souvent et peut donner tardivement des métastases.

Une femme de 51 ans a consulté en 1997 pour un nodule du bord gauche de langue non douloureux, qu'elle a découvert deux mois auparavant. L'aspect clinique ne présentait aucun signe spécifique ; le diagnostic a été réalisé grâce à l'analyse histopathologique et immunohistochimique de la pièce d'exérèse (positivité forte et diffuse pour la desmine, négativité pour l' $\alpha$-smooth muscle actin, la laminine, la cytokératine, le HHF-35 et la protéine S100). Ces éléments ont permis de conclure qu'il s'agissait d'un sarcome myofibroblastique de bas grade. La patiente a été contrôlée régulièrement pendant huit ans : il n'y a pas eu de récidive ni de métastase. (Med Buccale Chir Buccale 2008 ; $14: 239-244)$.

médecine buccale chirurgie buccale

VOL. $14, \mathrm{~N}^{\circ} 4$ 2008

page 239

mots clés: sarcome, myofibroblaste, langue

\section{SUMMARY}

Myofibroblastic sarcoma is an extremely rare malignant tumor of mesenchymal origin. It may develop in all tissues, but most frequently it occurs in head and neck soft tissues. This tumor, which evolution is relatively asymptomatic, can often recur but rarely metastasise.

A 51-year-old woman consulted in 1997 for a non-painful nodule that she noticed two months before, located on the left border of the tongue. The clinical aspect was not characteristic; diagnosis was made through anatomopathologic analysis completed by immunohistochemisty (strong and diffuse positivity for desmin, negativity for $\alpha$ smooth muscle actin, laminin, cytokeratin, HHF-35, and protein S-100). These results enabled to conclude to a low-grade myofibroblastic sarcoma. After an eight-year follow-up, no recurrence or metastase is observed. (Med Buccale Chir Buccale 2008 ; 14: 239-244).

key words: sarcoma, myofibroblast, tongue 
médecine

buccale

chirurgie

buccale

VOL. $14, \mathrm{~N}^{\circ} 4$ 2008

page 240
Les myofibroblastes sont des cellules d'origine mésenchymateuse, ayant des caractéristiques ultrastructurales communes avec les fibroblastes et les cellules musculaires lisses. Ils sont retrouvés dans quelques processus réactionnels (fasciite nodulaire, tissu de granulation) et dans des tumeurs bénignes (fibromatose, myofibroblastome). Le sarcome myofibroblastique est une tumeur maligne extrêmement rare et, ce n'est qu'avec la publication d'un nombre croissant de cas rapportés ces dernières années, qu'il a été reconnu comme une entité distincte du léïomyosarcome, du fibrosarcome et des autres tumeurs dérivées des cellules fusiformes. II peut toucher tous les organes et tous les tissus (y compris l'os), mais la majorité des cas concerne les tissus mous profonds, avec une localisation préférentielle pour la tête et le cou. On distingue plusieurs formes en fonction du grade de différenciation histologique: les myofibrosarcomes de bas grade et de grade intermédiaire, qui peuvent récidiver localement mais rarement métastaser, et le myofibrosarcome de haut grade ou pléomorphe dont les caractéristiques morphologiques et évolutives se rapprochent de celles de l'histiocytome fibreux malin. Le cas d'un myofibrosarcome de bas grade développé dans la langue chez une femme de 51 ans est présenté pour illustrer cette tumeur rare.

\section{OBSERVATION}

Une femme de 51 ans se présente en juin 1997 pour une tuméfaction indolore du bord antéro-latéral gauche de la langue, évoluant depuis environ deux mois. L'anamnèse révèle un antécédent de carcinome baso-cellulaire de l'aile droite du nez, traité chirurgicalement en 1994. Elle est en bon état général, elle ne prend aucun médicament et ne fume pas. Elle a consulté un mois auparavant un ORL pour le même motif ; il a réalisé un bilan sanguin dont les résultats étaient normaux et il a pratiqué une biopsie à la pince ; l'examen histopathologique n'a pas permis de proposer une orientation diagnostique.

L'examen exo-buccal est normal, on ne palpe pas d'adénopathie dans le territoire cervico-facial. L'examen endo-buccal montre un nodule sur le bord antéro-latéral gauche de la langue, bien circonscrit, mesurant environ $2 \mathrm{~cm}$ dans son grand axe, occupant toute l'épaisseur de la langue (Fig. 1). La muqueuse de revêtement est d'aspect normal, mais on observe une petite tumeur ressemblant à un botryomycome, développé sur le site de la biopsie effectuée 5 semaines auparavant. A la palpation, ce nodule de consistance élastique est légèrement douloureux.

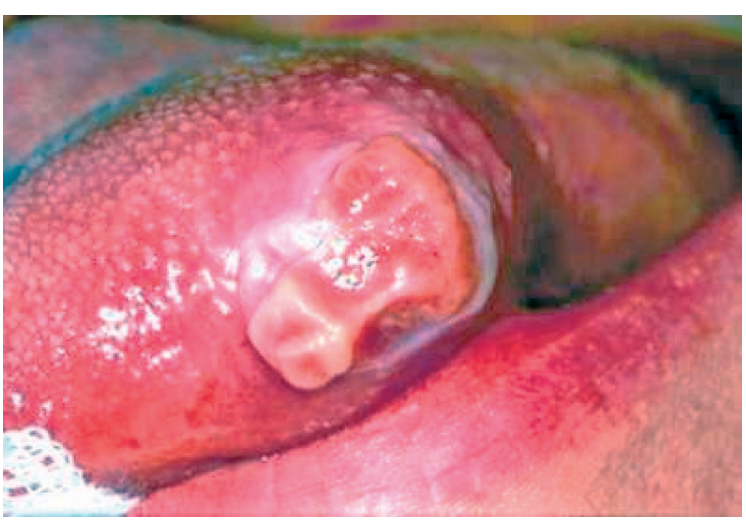

Figure 1 : Aspect de la tumeur lors de l'examen initial. Preoperative view.

En raison de la taille réduite de la tumeur et de son aspect limité sur l'IRM réalisé à la demande de l'ORL (Fig. 2), il a été décidé de procéder d'emblée à une exérèse in toto sous anesthésie locale.

La pièce d'exérèse mesure 1,6 x 1,4 x 1,1 cm. L'examen histopathologique montre un épithélium malpighien normal, interrompu par une ulcération correspondant au siège d'implantation du botryo-

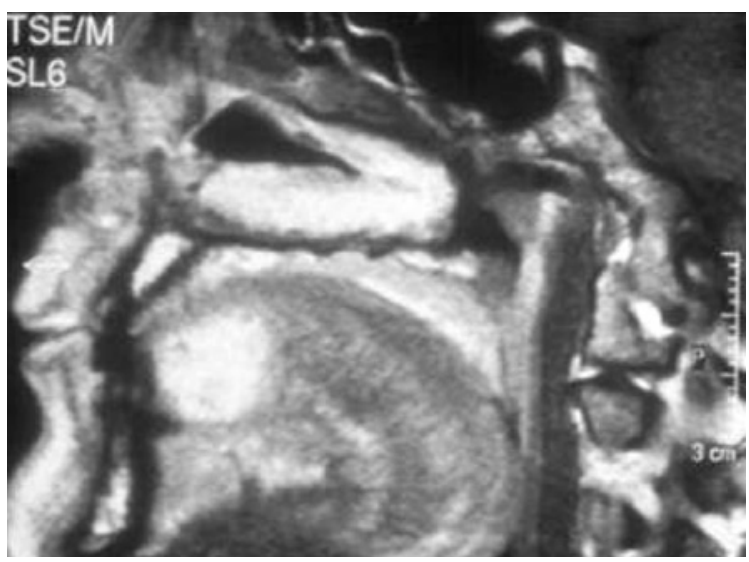

Figure 2 : IRM : lésion linguale arrondie, bien délimitée, occupant toute l'épaisseur de la langue mobile. MRI: well-limited,round image indicative of a tumor of the tongue occupying almost its entire body. 
mycome, séquelle de la première biopsie. En profondeur, le plan musculaire et le tissu graisseux adjacent sont envahis par une prolifération tumorale (Fig. 3) composée de cellules fusiformes indifférenciées, à noyau basophile et hyperchromatique ; certaines cellules comportent des atypies nucléaires (Fig. 4). L'analyse immunohistochimique montre une positivité forte et diffuse pour la desmine (Fig. 5) et

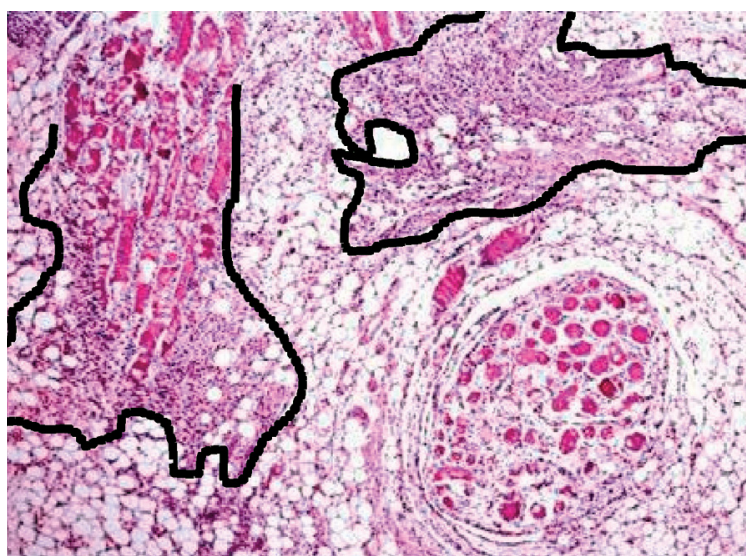

Figure 3 : Prolifération cellulaire formant des plages compactes s'insinuant entre les fibres musculaires et le tissu adipeux (faible grossissement $x 4$ ). Les deux principales sont entourées d'un trait.

Marked cellular proliferation surrounded between muscle fibres and adipose tissue, indicated by two doted lines (low power magnification $x 4$ ).

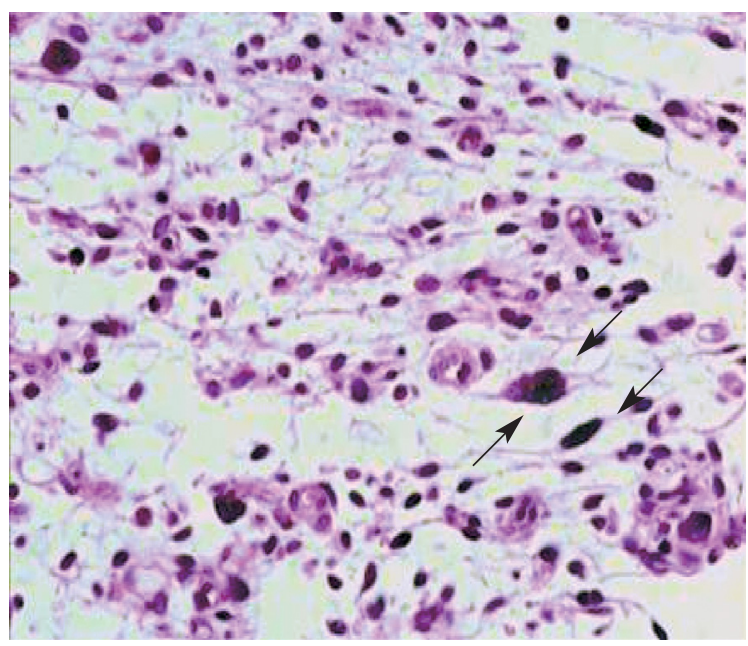

Figure 4 : Cellules à noyaux fusiformes, hyperchromatiques et atypiques (fort grossissement x 40), en particulier les trois indiqués par une flèche.

Cells with darkly stained nuclei (hyperchromatism), irregular (fusiform) with atypical (abnormal mitosis). Three nuclei are indicated with an arrow (high power magnification $x$ 40).

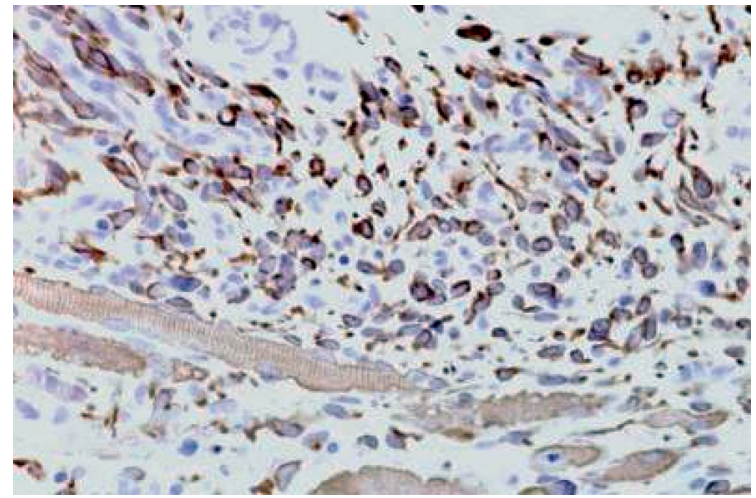

Figure 5 : Immunomarquage positif pour la desmine. Most tumour cells stained positive for desmine.

négative pour l' $\alpha$ - actine muscle lisse (Fig. 6), la laminine, la cytokératine, le HHF-35 et la protéine S100. Tous ces éléments orientent vers le diagnostic de sarcome myofibroblastique de bas grade.

Les marges de l'exérèse passant à un endroit en tissu tumoral, une exérèse complémentaire est proposée à la patiente, mais celle-ci refuse dans un premier temps toute réintervention. Ce n'est que trois mois plus tard, après la disparition de l'inflammation secondaire à l'exérèse chirurgicale, qu'elle est totalement convaincue de la persistance d'un nodule tumoral ; elle accepte alors la reprise chirurgicale proposée. L'examen histopathologique de la nouvelle pièce d'exérèse $(2,2 \times 1,2 \times 0,9 \mathrm{~cm})$ confirme bien la persistance d'un reliquat tumoral ; cette fois, les marges sont en tissu sain, sauf peut

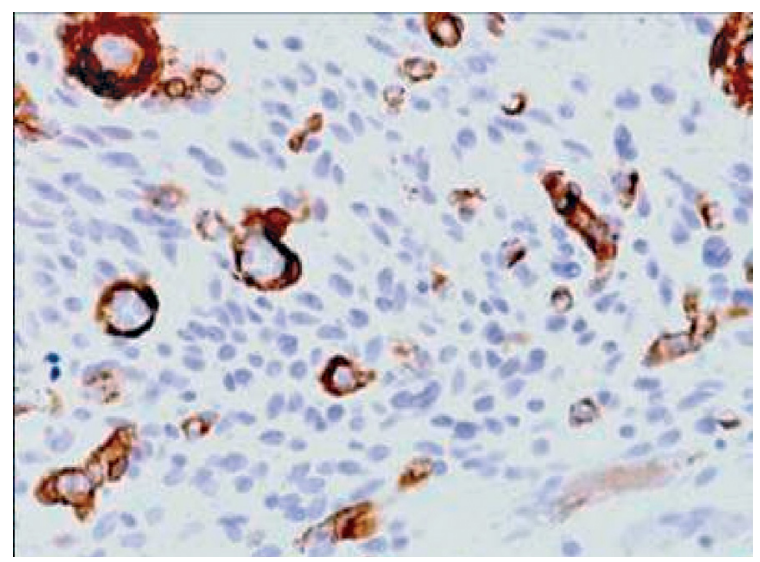

Figure 6 : Immunomarquage négatif pour l' $\alpha$-actine muscle lisse : seuls les vaisseaux sont marqués.

Tumor cells were not stained by $\alpha$-smooth muscle actin: only vessels are positive. médecine buccale chirurgie buccale

VOL. $14, \mathrm{~N}^{\circ} 4$ 2008

page 241 
être dans la région antéro-supérieure. Aucune autre intervention n'est programmée et, en accord avec la patiente, on opte pour des examens de contrôle réguliers et rapprochés. Après la reprise chirurgicale, l'amputation est assez conséquente et il existe une bride qui limite la mobilité linguale (Fig. 7). La disparition de l'inflammation et la réalisation d'exercices ont permis de retrouver une mobilité linguale presque normale (Fig. 8). En 2002, elle développe une tumeur desmoïde du fascia du biceps droit. Les examens de contrôle sont poursuivis jusqu'en 2005, et huit ans après l'exérèse il n'y a pas de récidive (Fig. 8) ni de métastase.

médecine buccale chirurgie buccale

VOL. $14, \mathrm{~N}^{\circ} 4$ 2008

page 242

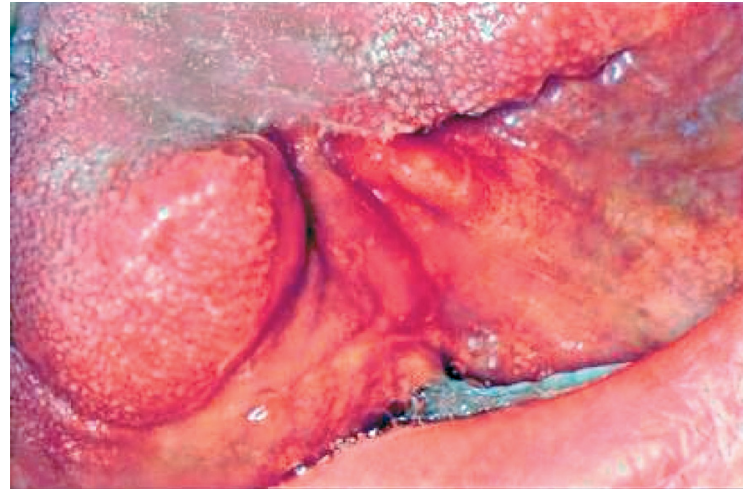

Figure 7 : Aspect postopératoire (6 semaines) : présence d'une bride limitant la mobilité linguale. Post-operative view (6 weeks).

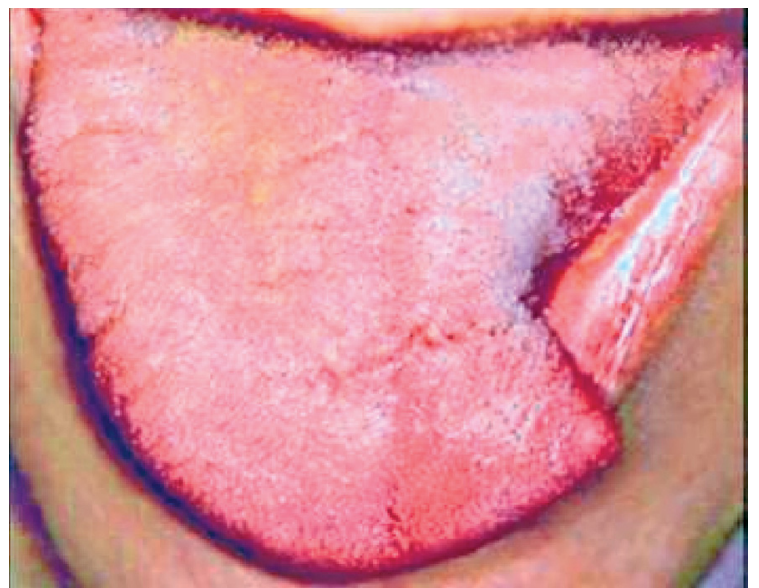

Figure 8 : Aspect postopératoire (8 ans) : l'amputation est toujours visible mais la langue a retrouvé une mobilité quasi normale.

Postoperative wiew (8 years).

\section{DISCUSSION}

Les sarcomes sont des affections relativement rares. Dans une étude rétrospective portant sur 45 ans et 11250 cancers de la région tête et cou, Gorsky et Epstein retrouvent 139 sarcomes $(1,24 \%)$, dont $16(0,14 \%)$ intéressaient les tissus mous de la cavité buccale [11]. Les sarcomes des tissus mous se développant à partir du tissu conjonctif, ils peuvent donc dériver du tissu fibreux, cartilagineux, adipeux, musculaire, vasculaire ou nerveux, en fonction de l'origine des cellules mésenchymateuses impliquées dans le processus tumoral.

Les myofibroblastes sont des cellules fusiformes ayant des caractéristiques qui les placent à michemin entre le fibroblaste et la cellule musculaire lisse. Comme ils possèdent la capacité de modifications fonctionnelles et phénotypiques, certains auteurs ont longtemps douté de l'existence d'une tumeur composée en grande partie ou en totalité de myofibroblastes [10]. Cela explique en partie le faible nombre de cas rapportés. Avant l'ère de la microscopie électronique, le myofibrosarcome était souvent confondu avec un fibrosarcome ou un léïomyosarcome ${ }^{[4,5,8]}$.

D'après l'étude de Mentzel (1998) portant sur 18 cas de sarcomes myofibroblastiques, l'âge moyen de survenue se situe vers 42 ans, et la répartition est égale entre les hommes et les femmes ${ }^{[13]}$. Le tableau clinique classique est celui de l'apparition d'une tuméfaction indolore, très rarement elle peut occasionner une gêne, sans autre manifestation. Sur ces 18 cas, 5 concernaient la cavité buccale, et 4 d'entre eux la langue. En microscopie standard, le sarcome myofibroblastique se présente comme une tumeur non encapsulée, parfois circonscrite, mais le plus souvent elle infiltre les tissus adjacents (musculaires ou graisseux). Son aspect microscopique ressemble à celui fibrosarcome : il est composé de cellules fusiformes et d'un stroma plus ou moins collagénique, qui est parfois le siège d'un infiltrat inflammatoire peu abondant. II existe des atypies nucléaires et l'activité mitotique est variable, mais il y a souvent des figures mitotiques anormales. L'étude ultrastructurale est certainement l'unique moyen de confirmer la différenciation myofibro- 
blastique mais, sur l'ensemble des cas de myofibrosarcomes rapportés dans la littérature, seule environ la moitié des cas a bénéficié d'une étude en microscopie électronique [14]. L'examen en microscopie électronique n'a pas été effectué pour le cas rapporté car l'aspect en microscopie standard était assez caractéristique : il présentait une similitude avec les autres cas publiés et, d'autre part, il ressemblait aux autres processus réactifs myofibroblastiques.

L'aspect clinique et les trois techniques paracliniques (la microscopie à balayage, l'immunohistochimie et la microscopie électronique décrites ci-dessus) permettent de démontrer sans équivoque l'origine myofibroblastique de ces tumeurs ${ }^{[8]}$. Le diagnostic des tumeurs myofibroblastiques doit préciser leur grade histologique puisqu'elles constituent un groupe avec des lésions ayant des comportements bien différents [3].

Une étude en immunohistochimie a été réalisée, dans le but de détecter les éventuels marqueurs de différenciation myoïde. Classiquement, il est admis que la positivité de l'immunoréaction pour l' $\alpha$-smooth muscle actin (SMA) constitue un bon argument en faveur du diagnostic du myofibrosarcome. Cependant, la négativité pour la SMA, comme dans le cas présenté, ne constitue pas un critère permettant d'écarter le diagnostic de myofibrosarcome car certains cas, confirmés par microscopie électronique, étaient négatifs pour la SMA [14]. Le myofibrosarcome peut exprimer ensemble ou séparément la SMA et la desmine: il peut être desmine positive/SMA négative ou desmine négative/SMA positive [9]. Dans l'étude de Mentzel (1998) portant sur 18 cas, il y a plus de cas positifs pour la desmine que pour la SMA [13] ; dans les lésions myofibroblastiques réactionnelles, on observe le contraire.

Le diagnostic différentiel du sarcome myofibroblastique inclut les autres lésions myofibroblastiques réactionnelles ou bénignes : la fasciite nodulaire, la fibromatose, le fibrosarcome ou le léïomyosarcome [7]. La distinction entre ces lésions repose sur la synthèse des données cliniques et paracliniques.

L'exérèse chirurgicale de la lésion représente le traitement de choix. Certains auteurs conseillent d'y associer une radiothérapie en cas d'exérèse incomplète ou de localisation non accessible chirurgicalement. La patiente présentait une localisation linguale facilement accessible permettant de réaliser une exérèse simple. L'exérèse initiale étant insuffisante, une reprise chirurgicale a été réalisée, et l'analyse histopathologique de la nouvelle pièce d'exérèse a montré que les marges étaient en tissu sain ; toutefois, un doute subsistait sur une zone très réduite localisée à la face profonde antéro-supérieur. En raison du bas grade de ce sarcome myofibroblastique et son très faible potentiel métastatique, il a été décidé avec la patiente de se limiter à une surveillance régulière et prolongée. Lors du dernier contrôle, effectué 8 ans après l'exérèse, il n'y avait aucun signe de récidive ni de métastases.

Le pronostic du sarcome myofibroblastique dépend du grade histologique et des limites de l'exérèse [12-18]. L'examen histopathologique doit être complété par une étude en immunohistochimie ; dans certains cas, surtout pour les myofibrosarcomes de haut grade, une étude complémentaire en microscopie électronique doit être réalisée. Les récidives des sarcomes myofibroblastiques de bas grade sont assez fréquentes, elles sont observées dans $44 \%$ des cas [14], mais les métastases sont très rares. Par contre le myofibrosarcome de haut grade ou pléomorphe a un pronostic beaucoup plus sévère, avec $29 \%$ de récidives, $71 \%$ de métastases ayant une évolution fatale dans $41 \%$ des cas [9]. L'analyse de I'ADN des cellules tumorales peut contribuer à l'évaluation du potentiel de récurrence et de métastase de la tumeur [3]. médecine

buccale chirurgie buccale

VOL. $14, \mathrm{~N}^{\circ} 4$ 2008

page 243 


\section{RÉFÉRENCES}

1 - Artopoulou II, Lemon JC, Clayman GL, Chambers MS. Stent fabrication for graft immobilization following wide surgical excision of myofibroblastic sarcoma of the buccal mucosa: a clinical report. J Prosthet Dent 2006 ; $95: 280-5$

2 - Bisceglia M, Tricarico N, Minenna P, Magro G, Pasquinelli G. Myofibrosarcoma of the upper jawbones: a clinicopathologic and ultrastructural study of two cases. Ultrastruct Pathol $2001 ; 25$ : 385-97.

3 - Biselli R, Boldrini R, Ferlini C, Boglino C, Inserra A, Bosman C. Myofibroblastic tumours: neoplasias with divergent behaviour. Ultrastructural and flow cytometric analysis. Pathol Res Pract 1999 ; 195 : 619-32.

4 - Eyden B.The myofibroblast: a study of normal, reactive and neoplastic tissues, with an emphasis on ultrastructure. Part 2 - tumours and tumour-like lesions. J Submicrosc Cytol Pathol 2005 ; 37 : 231-96.

5 - Eyden BP, Christensen L. Leiomyosarcoma versus myofibrosarcoma: observations and terminology. Ultrastruct Pathol $1993 ; 17: 231-9$.

médecine

buccale

chirurgie

buccale

VOL. $14, \mathrm{~N}^{\circ} 4$ 2008

page 244
10 - Fletcher CD. Myofibroblastic tumours: an update. Verh Dtsch Ges Pathol 1998 ; 82 : 75-82.

11 - Gorsky M, Epstein JB. Head and neck and intra-oral soft tissue sarcomas. Oral Oncol 1998 ; 34 : 292-6.

12 - Kraus DH, Dubner S, Harrison LB, Strong EW, Hajdu SI, Kher U, Begg C, Brennan MF.Prognostic factors for recurrence and survival in head and neck soft tissue sarcomas. Cancer $1994 ; 74$ : 697-702.

13 - Mentzel T, Dry S, Katenkamp D, Fletcher CD. Lowgrade myofibroblastic sarcoma: analysis of 18 cases in the spectrum of myofibroblastic tumors. Am J Surg Pathol 1998 ; 22 : 1228-38.

14 - Montgomery E, Goldblum JR, Fisher C. Myofibrosarcoma: a clinicopathologic study. Am J Surg Pathol $2001 ; 25$ : 219-28.

15 - Potter BO, Sturgis EM. Sarcomas of the head and neck. Surg Oncol Clin N Am $2003 ; 12$ : 379-417.

16 - Russell WO, Cohen J, Enzinger F, Hajdu SI, Heise H, Martin RG, Meissner W, Miller WT, Schmitz RL, Suit HD. A clinical and pathological staging system for soft tissue sarcomas. Cancer $1977 ; 40: 1562-70$.

17 - Smith DM, Mahmoud HH, Jenkins JJ 3rd, Rao B, Hopkins KP, Parham DM. Myofibrosarcoma of the head and neck in children. Pediatr Pathol Lab Med 1995 ; $15: 403-18$.

18 - Weber RS, Benjamin RS, Peters LJ, Ro JY, Achon O, Goepfert H. Soft tissue sarcomas of the head and neck in adolescents and adults. Am J Surg 1986 ; 152 : 38692. 